\title{
Check-list das Annonaceae do estado do Mato Grosso do Sul, Brasil
}

\author{
Adriana Quintella Lobão', Jenifer de Carvalho Lopes² \& Renato de Mello-Silva²
}

\author{
'Universidade Federal Fluminense. Instituto de Biologia, Departamento de Biologia Geral. R. Outeiro de São João Batista s/n. \\ CEP 24020-971. Niterói, RJ, Brasil. alobao@hotmail.com \\ 2Universidade de São Paulo. Instituto de Biociências, Departamento de Botânica, Herbário SPF. Rua do Matão, 277, \\ Edifício Sobre-as-Ondas. CEP 05508-090. São Paulo, SP, Brasil.
}

Recebido em 27.IX.2014.

Aceito em 22.VII.2016

DOI 10.21826/2446-8231201873s123

\begin{abstract}
RESUMO - Uma listagem das espécies de Annonaceae do estado do Mato Grosso do Sul é apresentada. Annonaceae possui 2.440 espécies, 385 no Brasil e 19 no estado do Mato Grosso do Sul, distribuídas em cinco gêneros, Annona L., Bocageopsis R.E.Fr., Duguetia A.St.-Hil., Unonopsis R.E.Fr. e Xylopia L. Annona sylvatica A.St.-Hil., Bocageopsis mattogrossensis (R.E.Fr.) R.E.Fr. e Duguetia glabriuscula (R.E.Fr.) R.E.Fr. são três novos registros para o Mato Grosso do Sul. Xylopia nitida Dunal não é mais listada entre as espécies de Annonaceae que ocorrem no estado. O número reduzido de espécies desta família no Mato Grosso do Sul deve-se provavelmente à predominância de áreas de Cerrado no estado, já que são mais frequentes em florestas tropicais de baixas altitudes.
\end{abstract}

Palavras-chave: diversidade, flora, Annona, Magnoliales, Xylopia.

ABSTRACT - Checklist of Annonaceae from Mato Grosso do Sul state, Brazil. A checklist of Annonaceae from Mato Grosso do Sul is presented. Annonaceae has 2,440 species, 385 in Brazil and 19 in the state of Mato Grosso do Sul with five genera, Annona L., Bocageopsis R.E.Fr., Duguetia A.St.-Hil., Unonopsis R.E.Fr., Xylopia L. Annona sylvatica A.St.-Hil., Bocageopsis mattogrossensis (R.E.Fr.) R.E.Fr. and Duguetia glabriuscula (R.E.Fr.) R.E.Fr. are three new records for Mato Grosso do Sul. Xylopia nitida Dunal is not pointed out between the species of Annonaceae from Mato Grosso do Sul. The low species number of this family in the state is probably due to the prevalence of Cerrado, since they are more frequent in lowland rain forests.

Keywords: diversity, flora, Annona, Magnoliales, Xylopia.

\section{INTRODUÇÃO}

Annonaceae é a maior família de Magnoliales (Chatrou et al. 2012) e uma das mais ricas em número de espécies entre as angiospermas, contando com 112 gêneros e 2.440 espécies (Couvreur et al. 2011). No Neotrópico está representada por aproximadamente 40 gêneros e 900 espécies (Chatrou et al. 2004), dos quais 29 gêneros e 385 espécies ocorrem no Brasil. A mais alta diversidade de espécies, 280, ocorre na região amazônica (Maas et al. 2012). A família é pantropical e pode ser reconhecida pelas folhas simples, alternas e dísticas, flor trímera, estames e carpelos numerosos e fruto apocárpico, pseudo-sincárpico ou sincárpico (Lobão et al. 2005).

Diversos levantamentos de Annonaceae no Brasil foram realizados nos últimos anos (Cruvinel et al. 2006, Dias \& Kinoshita 1996, Harley \& Simmons 1986, Kurtz \& Costa 1996, Lobão \& Mello-Silva 2007, Lobão et al. 2005, 2006 , 2012, Lopes 2012, Lopes \& Mello-Silva 2012, Maas et al. 2007, Mello-Silva 1993, 1997, Mello-Silva \& Pirani 2003, Mello-Silva \& Lopes 2011, Mello-Silva et al. 2012a, 2012b, Pontes 2009, Pontes \& Mello-Silva 2004, 2005, Pontes et al. 2004, Stannard 1995, Vattimo 1957 e Záchia \& Irgang
2004) mas alguns estados brasileiros, entre eles o Mato Grosso do Sul, ainda não possuem nenhum levantamento de Annonaceae. O presente "checklist", além de listar as espécies de Annonaceae, apresenta o atual estado do conhecimento da família no Mato Grosso do Sul.

$\mathrm{Na}$ área da sistemática, existem alguns grupos de estudo em Annonaceae. No Brasil, atuam os grupos da Universidade de São Paulo (USP) sob a coordenação do Prof. Dr. Renato de Mello-Silva e da Universidade Federal Fluminense (UFF) com a Profa. Dra. Adriana Quintella Lobão. No exterior, existem os grupos da Universidade de Wageningen, na Holanda, coordenado por Prof. Paul Maas e por Dr. Lars Chatrou; da Universidade de Viena, na Áustria, coordenado por Dr. Heimo Rainer e da Universidade de Ohio Wesleyan, nos Estados Unidos da América, coordenado por Dr. David Johnson.

No Brasil, a escassez de coletas e falta de trabalhos sistemáticos, principalmente na Amazônia, parecem ser lacunas no conhecimento geral das Annonaceae. No Mato Grosso do Sul, a lacuna mais básica é a falta de informação proporcionada pela falta de coletas amplas e gerais, que tornem as coleções de Annonaceae do estado mais significativas e conclusivas. 


\section{MATERIAL E MÉTODOS}

A listagem foi baseda em Maas et al. (2012) e nas bases de dados SBRJ e CRIA (2015) bem como nas coleções dos herbários ALCB, BHCB, BOTU, BR, CGMS, EAC, ESA, F, FLOR, FUEL, FURB, HCF, HPL, HSJRP, HUEFS, HUFU, IAC, INPA, MBM, RB, SP, SPF, SPFS, UB, UEC e UPCB, acrônimos segundo Thiers (2013).

\section{RESULTADOS E DISCUSSÃO}

Foram registrados cinco gêneros e 19 espécies de Annonaceae no Mato Grosso do Sul (Quadro 1). Levantamento anterior (Maas et al. 2012) contabiliza 18 espécies. O gênero com maior riqueza de espécies é Annona, com 12 espécies, seguido de Duguetia com três espécies, Xylopia com duas e Bocageopsis e Unonopsis com uma espécie cada. Não são encontradas espécies de Guatteria Ruiz et Pav., gênero frequente nos estados brasileiros próximos ao Mato Grosso do Sul, como Mato Grosso e Goiás, sendo Annona sylvatica, B. mattogrossensis e D. glabriuscula não registradas em seu levantamento. Xylopia nitida Dunal, citada em Maas et al. (2012) não é incluída na lista por falta de registros.

A elevada diversidade específica de Annona é consequência, em parte, da atual inclusão, em Annona, das espécies antes classificadas em Rollinia (vide Rainer 2007). Além disso, Mato Grosso do Sul possui diversas fitofissionomias que permitem tanto a ocorrência de espécies comuns no Cerrado, como A. coriacea, A. cornifolia, A. crassiflora, A. crotonifolia, A. dioica, A. glaucophylla e $A$. tomentosa, quanto espécies comuns em áreas florestadas, como A. cacans, A. emarginata e A. montana.

Das espécies levantadas, Annona cacans, A. montana e A. sylvatica ocorrem somente em Mata Atlântica; $A$. emarginata, Bocageopsis mattogrossensis, Unonopsis guatterioides e Xylopia emarginata ocorrem na Mata Atlântica e Cerrado, e A. coriacea, A. cornifolia, $A$. crassiflora, A. crotonifolia, A. dioica, A. glaucophylla, A. nutans, A. tomentosa, Duguetia furfuracea, $D$. glabriuscula, D. phaeoclados e X. aromatica ocorrem somente em Cerrado.

A diversidade específica do Mato Grosso do Sul é baixa em comparação com estados limítrofes. O Mato Grosso possui 60 espécies de Annonaceae, Goiás 31, Minas Gerais 58, Paraná 24 e São Paulo 31 espécies (Maas et al. 2012). Isto decorre, talvez, da grande extensão das áreas cobertas por Cerrado no Mato Grosso do Sul. Esta vegetação é relativamente pobre em espécies desta família que é composta basicamente por espécies arbóreas e arbustivas, sendo comum em áreas florestais como as Florestas Ombrófilas Densas. Também o esforço de coleta nesta região pode estar ligado número baixo de espécies encontradas, e este esforço precisa ser intensificado.

Das espécies amostradas, nenhuma é endêmica do estado. Entretanto, Annona glaucophylla e Duguetia phaeoclados são endêmicas dos Cerrados do Mato Grosso e Mato Grosso do Sul, onde poucos registros são conhecidos. Annona nutans apresenta distribuição restrita a três estados brasileiros, Mato Grosso, Mato Grosso do Sul e Minas Gerais (Maas et al. 2012). Nove espécies, Annona coriacea, A. crassiflora, A. dioica, A. montana, A. tomentosa, Duguetia furfuracea, Unonopsis guatterioides, Xylopia aromatica e $X$. emarginata são de ampla distribuição (Maas et al. 2012). Destas, D. furfuracea, $U$. guatterioides e $X$. aromatica ocorrem em grandes populações.

Dentre as espécies do Mato Grosso do Sul, nenhuma se encontra ameaçada de extinção no Brasil (MMA 2008). Entretanto, A. cacans e A. emarginata estão em perigo (EM) e vulnerável (VU), respectivamente,

Quadro 1. Espécies de Annonaceae no estado do Mato Grosso do Sul.

\begin{tabular}{|c|c|c|}
\hline Espécie & Voucher & Herbário \\
\hline Annona cacans Warm & U. Pastore 116 & $\mathrm{RB}$ \\
\hline A. coriacea Mart. & A. Amaral Jr. 08 & $\mathrm{RB}$ \\
\hline A. cornifolia A.St.-Hil. & R. L. Loureiro s.n. & RB 204351 \\
\hline A. crassiflora Mart. & I. A. Rodrigues 232 & $\mathrm{RB}$ \\
\hline A. crotonifolia Mart. & I. A. Rodrigues 216 & $\mathrm{RB}$ \\
\hline A. dioica A.St.-Hil. & L. C. Giordano 2749 & $\mathrm{RB}$ \\
\hline A. emarginata (Schltdl.) H.Rainer & G. Hatschbach 76040 & $\mathrm{RB}, \mathrm{SPF}$ \\
\hline A. glaucophylla R.E.Fr. & I. Cordeiro 1058 & SP, SPF \\
\hline A. montana Macfad. & G. A. Damaceno-Jr. 2556 & CGMS, SPF \\
\hline A. nutans (R.E.Fr.) R.E.Fr. & G.A. Damasceno Junior 3081 & CGMS, SPF \\
\hline A. sylvatica (A.St.-Hil.) Mart. & G.A. Damasceno Junior 3132 & CGMS, SPF \\
\hline A. tomentosa R.E.Fr. & I. Cordeiro 1082 & SP, SPF \\
\hline Bocageopsis mattogrossensis (R.E.Fr.) R.E.Fr. & R. Tsuji 2383 & HPL, SPF \\
\hline Duguetia furfuracea (A.St.-Hil.) Saff. & I. A. Rodrigues 329 & $\mathrm{RB}$ \\
\hline D. phaeoclados (Mart.) Maas \& H.Rainer & G.A. Damasceno Junior 2633 & CGMS, SPF \\
\hline D. glabriuscula (R.E.Fr.) R.E.Fr. & J. M. Siqueira s.n. & CGMS 4769 \\
\hline Unonopsis guatterioides (A.DC.) R.E.Fr. & G. Hatschbach 73366 & MBM, SPF \\
\hline Xylopia aromatica (Lam.) Mart. & J. P. P. Carauta 765 & $\mathrm{RB}$ \\
\hline X. emarginata Mart. & A. Amaral Jr. 236 & $\mathrm{RB}$ \\
\hline
\end{tabular}


no Rio Grande do Sul (Decreto Estadual 42.099 de 31 de dezembro de 2002). As espécies do Mato Grosso do Sul ainda não estão classificadas quanto a este critério, mas levando-se em conta ocorrência rara e as populações escassas, A. sylvatica, B. mattogrossensis, $D$. phaeoclados e D. glabriuscula, com menos de cinco registros conhecidos de cada uma, poderiam ser consideradas ameaçadas e, assim, possuírem elevado valor conservacionista no estado.

Grande parte do material de Annonaceae do Mato Grosso do Sul encontra-se no herbário CGMS (403 registros). Outras coleções significativas são encontradas nos herbários UEC (88 registros), RB (60) e MBM (57) e BR (55). Segundo o banco de dados CRIA (2015), os principais acervos desta família são: Herbário do Instituto Nacional de Pesquisa da Amazônia (INPA) com 5774 registros, Instituto de Pesquisas Jardim Botânico do Rio de Janeiro (RB) com 4934, Jardim Botânico de New York (NY) com 4689, Jardim Botânico do Missouri (MOBOT) com 2184, Universidade Estadual de Campinas (UEC) com 1483, Instituto de Botânica de São Paulo (SP) com 1532, Museu Botânico Municipal de Curitiba (MBM) com 1498, Centro de Pesquisas do Cacau na Bahia (CEPLAC) com 1391, Universidade de Brasília (UB) com 1252 e Universidade Estadual de Feira de Santana (HUEFS) com 1038. Outra coleção com um importante acervo é o Herbário do Instituto de Biociências da Universidade de São Paulo (SPF). Por ser um dos centros de pesquisa em Annonaceae, a coleção do SPF possui diversos exemplares principalmente de procedência da Mata Atlântica e do cerrado.

Diversos trabalhos revisionais de gêneros neotropicais de Annonaceae foram publicados desde o início do projeto de sistemática liderado por Dr. Paul Maas (Maas 1983). A maioria dos gêneros de ocorrência no Brasil já foram revistos e os que ainda não o foram, como Annona e Xylopia, já estão em processo de revisão por pesquisadores estrangeiros. Apesar disso, há diversas espécies de ocorrência no Brasil que precisam ser melhor estudadas quanto a sua delimitação e taxonomia.

A filogenia das Annonaceae foi reconstruída nos trabalhos de Doyle \& Le Thomas (1994, 1996), Doyle et al. (2000) e Richardson et al. (2004) e recentemente uma nova classificação para a família foi proposta (Chatrou et al. 2012). No entanto, há poucos estudos sobre a biogeografia e filogenia dos gêneros neotropicais, como os estudos desenvolvidos por Erkens et al. (2007a, 2007b) com Guatteria. Assim, as perspectivas de pesquisa para os próximos anos são a reconstrução da filogenia dos gêneros neotropicais e o estudo de sua biogeografia e o aprofundamento do estudo da diversidade desta família no Brasil.

\section{AGRADECIMENTOS}

Agradecemos à Fundação de Amparo a Pesquisa do Estado de São Paulo pela bolsa de doutorado ao segundo autor e ao Conselho Nacional de Desenvolvimento
Científico e Tecnológico pela bolsa de produtividade ao terceiro autor.

\section{REFERÊNCIAS}

Chatrou, L.W., Rainner, H. \& Maas, P.J.M. 2004. Annonaceae. In Flowering plants of the Neotropics (N. Smith, N., S.A. Mori, A. Henderson, D.W. Stevenson \& S.V. Heald, eds.). Princeton University Press, The New York Botanical Garden, Princeton, New York, p. 18-20.

Chatrou, L.W., Pirie, M.D., Erkens, R.H.J., Couvreur, T.L.P., Neubig, K.M.J., Abbott, R., Mols, J.B., Maas, J.W., Saunders, R.M.K. \& Chase, M.W. 2012. A new subfamilial and tribal classification of the pantropical flowering plant family Annonaceae informed by molecular phylogenetics. Botanical Journal of the Linnean Society 169(1): 5-40.

Couvreur, T.L.P., Pirie, M.D., Chatrou, L.W., Saunders, R.M.K., SU, Y.C.F., Richardson, J.E. \& Erkens, R.H.J. 2011. Early evolutionary history of the flowering plant family Annonaceae: steady diversification and boreotropical geodispersal. Journal of Biogeography 38: 664-680.

Centro de Referência em Informação Ambiental - CRIA 2015. SpeciesLink. Disponível em: http://www.splink.org.br/index. Acessado em 23.01.2013.

Cruvinel, S.R.C., Rodrigues, W.A. \& Rizzo, J.A. 2006. Flora dos Estados de Goiás e Tocantins: Annonaceae, Coleção Rizzo v. 35. Gráfica e Editora Vieira, Goiânia. 60p.

Dias, M.C. \& Kinoshita, L.S. 1996. Flora fanerogâmica da reserva do Parque Estadual das Fontes do Ipiranga (São Paulo, Brasil). 3 Annonaceae. Hoehnea 23(2): 107-111.

Doyle, J.A., Bygrave, P. \& Le Thomas, A. 2000. Implications of molecular data for pollen evolution in Annonaceae. In Pollen and Spores: morphology and biology (M.M. Harley, C.M. Morton \& S. Blackmore, eds.). Royal Botanic Gardens, Kew, p. 259-284

Doyle, J.A. \& Le Thomas, A. 1994. Cladistic analysis and pollen evolution in Annonaceae. Acta Botanica Gallica 141(2): 149-170.

1996. Phylogenetic analysis and character evolution in Annonaceae. Bulletin du Muséum National d'Histoire Naturelle Section B 18: 279-334.

Erkens, R.H.J., Chatrou, L.W., Koek-Noorman, J., Maas, J.W. \& Maas, P.J.M. 2007a. Classification of the large widespread genus of Neotropical trees, Guatteria (Annonaceae) and its three satellite genera Guatteriella, Guatteriopsis and Heteropetalum. Taxon 56(3): 757-774.

Erkens, R.H.J., Chatrou, L.W., Maas, J.W. Van Der Niet, T., Savolainen, V. 2007b. A rapid diversification of rainforest trees (Guatteria; Annonaceae) following dispersal from Central into South America. Molecular Phylogenetics and Evolution 44: 399-411.

Kurtz, B.C. \& Costa, A. 1996. Annonaceae. In Reserva Ecológica de Macaé de Cima, Nova Friburgo, RJ. Aspectos florísticos das plantas vasculares (M.P.M. Lima \& R.R. Guedes-Bruni, orgs.). Jardim Botânico do Rio de Janeiro. Rio de Janeiro, p. 41-56.

Harley, R.M. \& Simmons, N.A. 1986. Annonaceae. In Florula of Mucugê, Chapada Diamantina, Bahia, Brazil: a descriptive check-list of a campo rupestre area (R.M. Harley \& N.A. Simmons). Royal Botanical Gardens. Kew, p. 17-18.

Jabot. Disponível em: www.jbrj.gov.br/jabot/. Acessado em: 19.05.2016. JBRJ - Instituto de Pesquisas Jardim Botânico do Rio de Janeiro. Jabot - Banco de Dados da Flora Brasileira. Disponível em: [http://www. jbrj.gov.br/jabot]. Acessado em 22.07.2016.

Lobão, A.Q., Araujo, D.S.D. \& Kurtz, B.C. 2005. Annonaceae das Restingas do Estado do Rio de Janeiro, Brasil. Rodriguésia 56(87): 85-96.

Lobão A.Q., Forzza, R.C. \& Mello-Silva, R. 2006. Annonaceae da Reserva Biológica do Grama, Descoberto, Minas Gerais, Brasil, com uma nova espécie, Unonopsis bauxitae. Rodriguésia 57(1): 137-147.

Lobão, A.Q. \& Mello-Silva, R. 2007. Guatteria (Annonaceae) do Estado do Rio de Janeiro. Rodriguésia 58(4): 859-884.

Lobão, A.Q., Mello-Silva, R. \& Forzza, R.C. 2012. Guatteria (Annonaceae) da Floresta Atlântica. Rodriguésia: 63(4). Disponível em: http:// rodriguesia.jbrj.gov.br/. Acessado em 19.02.2013. 
Lopes, J.C. 2012. Annonaceae da Reserva Natural Vale, Linhares, Espírito Santo. Dissertação 132 f., Universidade de São Paulo.

Lopes, J.C. \& Mello-Silva, R. 2012. Annonaceae do Parque Estadual de Ibitipoca, Minas Gerais. Boletim de Botânica da Universidade de São Paulo 30(2): 157-164.

Maas, P.J.M. 1983. Project Systematics of Annonaceae. Taxon 32(3): 528-529.

Maas, P.J.M., Kamer, H.M.-van, Junikka, L., Mello-silva, R. \& Rainer, H. 2002. Annonaceae from Central-eastern Brazil. Rodriguésia 52(80): 61-94.

Maas, P.J.M., Maas, H. \& Miralha, J. M. S. 2007. Flora da Reserva Ducke, Amazonas, Brasil: Annonaceae. Rodriguésia 58 (3): 617-662.

Maas, P., Rainer, H., Lobão, A.Q. 2012. Annonaceae. In Lista de Espécies da Flora do Brasil. Jardim Botânico do Rio de Janeiro. Disponível em: http://floradobrasil.jbrj.gov.br/2012/FB110219. Acessado em 06.11.2012.

Mello-Silva, R. 1993. Annonaceae. Flora Fanerogâmica da Ilha do Cardoso 3: 43-57.

1997. Annonaceae. In Mapeamento da cobertura vegetal e listagem das espécies ocorrentes na área de proteção ambiental de Cairuçu, município de Parati, RJ (M.C.M. Marques, ed.). Jardim Botânico do Rio de Janeiro. Rio de Janeiro, p. 52-53.

Mello-Silva, R. \& Lopes, J.C. 2011. Checklist of Spermatophyta of the São Paulo State, Brazil: Annonaceae. Biota Neotropica 11: 193-390.

Mello-Silva, R., Lopes, J.C \& Pirani, J.R. 2012a. Flora da Serra do Cipó, Minas Gerais: Annonaceae. Boletim de Botânica da Universidade de São Paulo 30(1): 37-56.

Mello-Silva, R., Lopes, J.C. \& Rainer, H. 2012b. Annonaceae. Flora das Caatingas do Rio São Francisco. In Flora das Caatingas do Rio São Francisco (J.A. Siqueira-Filho, org.). Andrea Jakobsson, Rio de Janeiro, p. 462.

Mello-Silva, R. \& Pirani, J.R. 2003. Flora de Grão-Mogol, Minas Gerais: Annonaceae. Boletim de Botânica da Universidade de São Paulo 21(1): $67-72$.
Ministério do Meio Ambiente - MMA 2008. Disponível em: http://www.mma.gov.br/estruturas/ascom_boletins/_ arquivos/83 19092008034949.pdf. Acessado em 2.2013.

Pontes, A.F. 2009. Annonaceae. In Flora de Mirandiba-PE. (M. Alves, M.F. Araújo, J.R. Maciel \& S. Martins, orgs). Associação Plantas do Nordeste, Recife, v. 1, p. 59-60.

Pontes, A.F., Barbosa, M.R.V. \& Maas, P.J.M. 2004. Flora Paraibana: Annonaceae Juss. Acta Botanica Brasilica 18(2): 281-293.

Pontes, A.F. \& Mello-Silva, R. 2004. Annonaceae. In Flora do Distrito Federal, Brasil (T.B. Cavalcanti \& A.E. Ramos, eds.). Embrapa Recursos Genéticos e Biotecnologia. Brasília, v. 3, p. 21-48.

. 2005. Annonaceae do Parque Estadual da Serra da Canastra, Minas Gerais, Brasil. Boletim de Botânica da Universidade de São Paulo 23(1): 71-84.

Rainer, H. 2007. Monographic studies in the genus Annona L. (Annonaceae): Inclusion of the genus Rollinia A.St.-Hil. Annalen des Naturhistorischen Museums in Wien, B 108: 191-205.

Richardson, J.E., Chatrou, L.W., Mols, J.B., Erkens, R.H.J. \& Pirie, M.D. 2004. Historical biogeography of two cosmopolitan families of flowering plants: Annonaceae and Rhamnaceae. Philosophical Transactions of the Royal Society B 359: 1495-1508.

Stannard, B.L. 1995. Annonaceae. In Flora de Pico das Almas, Chapada Diamantina, Bahia, Brasil (B.L. Stannard, ed.). Royal Botanic Gardens. Kew, p. 126-128.

Thiers, B. [continuously updated]. Index Herbariorum: A global directory of public herbaria and associated staff. New York Botanical Garden's Virtual Herbarium. Disponível em: http://sweetgum.nybg.org/ih/. Acessado em 23.01.2013.

Vattimo, I. 1957. Annonaceae. Flora do Itatiaia-I. Rodriguésia 20(32): 42-51.

Záchia, R.A. \& Irgang, B.E. 2004. A família Annonaceae no Estado do Rio Grande do Sul, Brasil. Pesquisas Botânica 55: 7-127. 